\title{
Carlo Schmid et la puissance d'occupation française dans le Wurtemberg durant l'immédiat après- guerre : collaboration et confrontation entre deux espaces publics
}

Nadine Willmann

\section{(2) OpenEdition \\ Journals}

Édition électronique

URL : https://journals.openedition.org/allemagne/397

DOI : 10.4000/allemagne.397

ISSN : 2605-7913

Éditeur

Société d'études allemandes

Édition imprimée

Date de publication : 28 décembre 2016

Pagination : 289-304

ISSN : 0035-0974

Référence électronique

Nadine Willmann, «Carlo Schmid et la puissance d'occupation française dans le Wurtemberg durant l'immédiat après-guerre : collaboration et confrontation entre deux espaces publics ", Revue

d'Allemagne et des pays de langue allemande [En ligne], 48-2 | 2016, mis en ligne le 28 décembre 2017, consulté le 21 mai 2021. URL : http://journals.openedition.org/allemagne/397 ; DOI : https://doi.org/ 10.4000/allemagne.397 


\section{Carlo Schmid et la puissance d'occupation française dans le Wurtemberg durant l'immédiat après-guerre: collaboration et confrontation entre deux espaces publics}

- Nadine Willmann*

Né en 1896 à Perpignan de mère française, l'intellectuel et homme politique Carlo Schmid, dont la famille s'installa à Stuttgart dès 1908, était parfaitement bilingue. Ses études de droit international à Tübingen le menèrent jusqu'à l'habilitation, mais son rejet du national-socialisme le cantonna dans une activité de chargé de cours sous le III $^{\mathrm{e}}$ Reich. Sa formation, ainsi que ses fonctions de conseiller administratif militaire pour l'occupation à Lille, pendant la guerre, lui conférèrent une expérience du droit de l'occupation qui lui fut largement profitable après 1945. À cette époque, l'épreuve du national-socialisme et le sentiment d'avoir porté par sa passivité une part de responsabilité le persuadèrent de s'engager politiquement dans l'édification du nouvel État ${ }^{(1)}$.

Sa pratique du droit de l'occupation, conjuguée à ses origines, généra une relation de confiance avec l'occupant français, qui était confortée par son attitude, connue de celui-ci, de soutien à la population de Lille pendant la guerre ${ }^{(2)}$. Toutefois, la France sortait d'une occupation très rude, si bien que la politique de l'occupant était déterminée par l'impératif d'empêcher à jamais son voisin de déclarer à nouveau une guerre. De ce fait, la puissance d'occupation s'avéra intransigeante sous certains aspects et les rapports de Carlo Schmid avec elle se révélèrent une combinaison de collaboration et de confrontation. Nous analyserons cette relation dans une perspective chronologique, au fil des différentes fonctions gouvernementales exercées par Carlo Schmid dans le

* PRAG et docteur en études allemandes, Institut d'études politiques de Strasbourg, EA 1341 Études germaniques.

1 Gerhard Hirschler, Carlo Schmid und die Gründung der Bundesrepublik: eine politische Biographie, Bochum, Studienverlag Brockmeyer, 1986, p. 21-22.

2 Hellmuth Auerbach, «Die politischen Anfänge Carlo Schmids - Kooperation und Konfrontation mit der französischen Besatzungsmacht 1945-1948», Vierteljahrshefte für Zeitgeschichte, 4 (1988), p. 600 . 
Wurtemberg, et montrerons comment, après s'être forgé une place dans l'espace public allemand, Schmid pénètre dans l'espace public français et tente d'articuler les positions et les intérêts des deux espaces publics.

Nous entendons par conséquent la notion d'espace public comme l'espace de l'action gouvernementale, le lieu de la rencontre entre les différents acteurs gouvernementaux, dont les contacts sont scrupuleusement commentés par la presse et attentivement suivis par l'opinion publique. Cet espace public, ou plus exactement, ici, ces espaces publics - allemand et français - influent directement sur le quotidien de la population et, à ce titre, appartiennent à tous, dans la mesure où les citoyens s'approprient la sphère publique contrôlée par l'autorité pour la transformer en une sphère où la critique s'exerce contre le pouvoir de l'État, dans l'acception de Habermas. Ainsi l'action de Schmid dans sa relation avec l'occupant fait-elle tantôt l'objet d'éloges, tantôt l'objet de réprobation de la part de l'opinion publique.

\section{Accès à un rôle dans l'espace public allemand}

Les premières semaines après la guerre

De retour en Allemagne, Carlo Schmid ne tarde pas à accéder à une place de premier plan dans l'espace public allemand et il est bientôt reconnu comme une notabilité de premier plan à Tübingen puis dans le Wurtemberg. Et d'emblée, il acquiert cette notoriété sur fond de relation avec les Français et avec leur soutien, donc dans le cadre de la cohabitation des deux espaces publics. Dès les premiers jours de l'occupation, il se rend à la mairie de Tübingen, où il réside, pour faire l'intermédiaire entre les employés et l'occupant, qui, satisfait de sa mission de médiateur, le dispense bientôt du service de travail obligatoire ${ }^{(3)}$. En revanche, il n'est pas épargné par l'obligation de loger des soldats, notamment des Marocains qui utilisent son manuscrit sur Machiavel comme papier toilette $^{(4)}$. En avril 1945, il est arrêté par les Français, soupçonné d'être un Werwolf (loup-garou), un de ces francs-tireurs rassemblés par les SS à la fin de la guerre pour commettre des actes de sabotage contre les troupes d'occupation. Maltraité, il est profondément offensé. Il est bientôt libéré suite à la découverte de sa traduction de Baudelaire, qui infirme tout soupçon ${ }^{(5)}$.

Le même mois, il prend une initiative qui le propulse en première ligne de l'espace public de Tübingen: il rejoint le Bloc antifasciste, fondé par des sociaux-démocrates et des communistes, seul forum politique existant dans la cité, salué par les Français, qui vise à réunir des personnes susceptibles de contribuer à l'édification d'institutions démocratiques ${ }^{(6)}$. Sous sa houlette, l'organisation se transforme bientôt en l'Association démocratique, dont il s'évertue à tempérer le poids des communistes en ralliant des démocrates du centre ${ }^{(7)}$, afin de s'adresser à une population plus large et de ne pas effrayer les Français. En peu de temps, Schmid devient ainsi la personnalité

3 Petra Weber, Carlo Schmid: 1896-1979 - eine Biographie, Munich, Beck, 1996, p. 192.

4 G. Hirschler, Carlo Schmid und die Gründung der Bundesrepublik (note 1), p. 27.

P. Weber, Carlo Schmid: 1896-1979 (note 3), p. 192.

Carlo Schmid, Erinnerungen, Munich, Wilhelm Goldmann, 1981, p. 220.

Edgar Wolfrum, Französische Besatzungspolitik und deutsche Sozialdemokratie. Politische Neuansätze in der «vergessenen Zone» bis zur Bildung des Südweststaates 1945-1952, Düsseldorf, Droste, 1991, p. 60. 
dominante de l'association et, à travers son activité de médiateur entre la ville et l'occupant, de Tübingen ${ }^{(8)}$. Du fait de son français parfait, il entretient rapidement d'étroites relations avec les officiers français, particulièrement avec Gonzague Corbin de Mangoux, et il est bientôt dénoncé comme «l'homme des Français ». Néanmoins, la plupart des habitants apprécient ses efforts, qui visent à atténuer les rigueurs de l'occupation $^{(9)}$.

Avec l'Association démocratique était né un conseil municipal officieux, qui, débattant des urgences concrètes, jouait un rôle central dans l'espace public. De ses rangs émergèrent de nombreux titulaires de postes dans l'administration communale et régionale ${ }^{(10)}$. En mai 1945, Schmid obtient de l'occupant l'autorisation pour l'Association de nommer un conseil municipal provisoire, dont il est élu président, mais quelques jours plus tard, le commandement central français de Baden-Baden dissout l'assemblée ${ }^{(11)}$. Il estime en effet que la reconstruction doit s'effectuer du haut vers le bas et que les Allemands doivent être préalablement éduqués à la démocratie ${ }^{(12)}$. Dès lors, les jours de l'Association démocratique sont comptés, du fait des conflits internes et de la fondation de partis qui se profile.

\section{Ministre à Stuttgart}

Le rôle de Schmid dans l'espace public allemand s'étend alors à l'ensemble du Wurtemberg, toujours avec l'aval de l'occupant: le gouvernement militaire français doit rapidement avoir prévu de lui attribuer un poste dans une administration du Land, et l'Allemand est déterminé à s'investir, voulant empêcher que les Français fassent du Wurtemberg une colonie exploitée. Au terme d'une course avec l'armée américaine, les Français étaient parvenus à occuper Stuttgart et chargèrent son maire de nommer, en juin 1945, neuf directeurs régionaux (Landesdirektoren), l'équivalent de ministres. Mécontents du choix pour le ministre du Culte et de l'Éducation, ils imposent Schmid, qui, s'étant engagé pour la réorganisation de l'université de Tübingen, leur paraît la personnalité idoine ${ }^{(13)}$. C'est donc explicitement grâce à une intervention d'acteurs de l'espace public français que Schmid peut élargir son champ d'action dans l'espace public allemand.

Comme les Français, ce dernier accorde à l'éducation une portée fondamentale pour l'établissement de la démocratie et la politique scolaire qu'il met en place se révèle fondatrice pour le Wurtemberg ${ }^{(14)}$. Il débarrasse l'école des nationaux-socialistes trop zélés et supervise la publication de nouveaux manuels ${ }^{(15)}$. À l'instar du gouvernement militaire, il se prononce pour un principe de sélection, redoutant la formation d'un

8 H. Auerbach, «Die politischen Anfänge Carlo Schmids» (note 2), p. 600.

9 P. Weber, Carlo Schmid: 1896-1979 (note 3), p. 193.

10 G. Hirschler, Carlo Schmid und die Gründung der Bundesrepublik (note 1), p. 24-25.

11 Frank RABerg, «Wie Carlo Schmid in die Politik kam. Vom Landesdirektor in Stuttgart zum provisorischen Regierungschef in Tübingen», in: Gerhard TADDEy (dir.), Carlo Schmid - Mitgestalter der Nachkriegsentwicklung im deutschen Südwesten, Stuttgart, Kohlhammer, 1997, p. 12-13.

12 P. Weber, Carlo Schmid: 1896-1979 (note 3), p. 194.

13 Ibid., p. 202-203.

14 Ibid., p. 205.

15 F. RAberg, «Wie Carlo Schmid in die Politik kam» (note 11), p. 15. 
prolétariat académique, foyer du nationalisme, et voulant promouvoir une nouvelle élite ${ }^{(16)}$. Avec le retrait des Français de Stuttgart échoient le mandat de l'administration du Land et celui de Schmid, qui est toutefois nommé conseiller d'État (Staatsrat) du gouvernement de Wurtemberg-Bade, ce qui lui permet de continuer à assister aux séances du cabinet à Stuttgart et de devenir le médiateur entre Stuttgart et Tübingen, entre le Wurtemberg-Bade et le Wurtemberg-Hohenzollern, entre la zone américaine et la zone française ${ }^{(17)}$.

\section{Pénétration de l'espace public français}

\section{Ministre et chef de gouvernement en Wurtemberg-Hohenzollern}

En effet, le Wurtemberg a été séparé par l'occupation, au gré de l'entrée des armées, par une ligne de démarcation: "Cela nous a cruellement touchés, parce que pour nous le Wurtemberg n'est pas un simple district administratif [...], mais notre plus étroite patrie; [...] ce pays entre Neckar et Danube est pour nous une réalité vivante [...], c'est justement dans son intégralité intacte un élément dont la mutilation nous laisserait plus que démunis, à savoir comme d'autres que ceux que de nature nous sommes et voulons être [...]» ${ }^{(18)}$ écrit-il à l'époque. Toutefois, il ajoute que les Wurtembergeois ont reçu des puissances d'occupation l'assurance qu'elles veulent considérer le Land comme une unité politique, économique, culturelle et juridique.

Lorsque Schmid reconnaît l'inanité de ses efforts pour dissuader les Français de détacher le Wurtemberg-Hohenzollern de Stuttgart, il prend l'initiative et négocie avec le gouverneur Guillaume Widmer et son directeur de cabinet Corbin de Mangoux les conditions de création de l'autorité administrative ${ }^{(19)}$. À partir de ce moment, il s'efforce de pénétrer l'espace public français afin d'en tirer le plus de bénéfice possible pour l'espace public allemand et de cantonner ses intrusions dans le registre de l'acceptable. Il est clair qu'il sera le dirigeant de la future entité, ce qu'il doit, selon Widmer, à son origine, sa formation et son attitude à Lille ${ }^{(20)}$. En octobre 1945 se réunit le secrétariat d'État (Staatssekretariat) pour le Wurtemberg-Hohenzollern, sous la direction du conseiller d'État Schmid, directeur régional (c'est-à-dire ministre) pour la Justice et le Culte, l'Éducation et l'Art, ces différentes appellations étant instaurées par ce dernier. En s'engageant dans l'espace public français, Schmid conforte par conséquent sa fonction dans l'espace public allemand.

Il se souvient de ses résolutions: «acceptation du fait que l'autorité suprême repose entre les mains du gouvernement militaire, mais conscience que ceci ne dispense pas les responsables de notre Land du devoir, en cas de danger pour les intérêts vitaux

16 P. Weber, Carlo Schmid: 1896-1979 (note 3), p. 207.

17 F. Raberg, «Wie Carlo Schmid in die Politik kam» (note 11), p. 16.

18 «Es hat uns so bitter weh getan, weil W. für uns nicht ein bloßer Verwaltungsbezirk [...], sondern unser engeres Vaterland; [...] dieses Land an Neckar u Donau ist uns eine lebendige Wirklichkeit [...], es ist für und gerade in seiner ungebrochenen Ganzheit ein Element, dessen Versehrung uns mehr als arm zurücklassen würde, nämlich als andere als wir vom Wesen her sind und sein wollen [...]", Karl SснміD, «Gedanken zum Aufbau der Verwaltung», in: Karl Schмid, Die Forderung des Tages, Stuttgart, Klett, 1946, p. 81-82.

19 F. Raberg, «Wie Carlo Schmid in die Politik kam» (note 11), p. 17.

20 H. Auerbach, «Die politischen Anfänge Carlo Schmids» (note 2), p. 602. 
de la population [...], d'opposer une résistance tant à des ordres particuliers qu'à la politique générale de la puissance d'occupation» ${ }^{(21)}$. D’emblée, donc, il sollicite une opposition de l'espace public allemand si le poids de l'espace public français devait devenir trop accablant. De son côté, Widmer certifie que cette administration peut être assurée du soutien du gouvernement militaire, mais qu'elle est soumise pleinement à ses directives ${ }^{(22)}$. Schmid situe l'administration du Wurtemberg du Sud à un niveau inférieur à Stuttgart, car l'objectif reste l'unité du Land et le secrétariat d'État se considère comme une institution transitoire ${ }^{(23)}$.

Schmid s'entend à gagner pour le secrétariat d'État des personnalités remarquables, si bien que le Wurtemberg-Hohenzollern dispose d'un gouvernement performant bien avant le reste de la zone française ${ }^{(24)}$, et ce gouvernement dépasse le cadre d'une administration obtempérant aux ordres pour élargir, à l'initiative de Schmid, sa marge de manœuvre ${ }^{(25)}$. Ce dernier a donc su user de sa connaissance de l'espace public français pour en borner l'influence et sauvegarder une part croissante à l'espace public allemand. Le gouverneur Widmer, impressionné, félicite Schmid, qui pour sa part est reconnaissant à l'occupant de ne pas exiger une épuration de l'administration aussi radicale que les Américains. Lors des séances du directoire, Schmid est décrit comme «primus supra pares», qui fixe les lignes directrices de la politique ${ }^{(26)}$.

\section{Les dossiers politiques de prédilection de Carlo Schmid}

On peut distinguer quatre dossiers politiques de prédilection de Schmid: la politique de dénazification, le système scolaire, le système universitaire et les liens avec les sous-préfets (Landräte). Sous l'égide de Schmid, le système de dénazification se développe de manière exemplaire. Au printemps 1946, il est clair que les conclusions des commissions d'enquête des districts s'expriment de façon très inégale. Schmid met cette constatation à profit pour présenter en mai à Widmer l'ébauche d'une directive, qui, approuvée par le gouvernement militaire, a bientôt force de loi ${ }^{(27)}$. Il anticipe ainsi un changement d'orientation de la politique de l'occupant qui voulait exclure du service public $50 \%$ des fonctionnaires ayant été inscrits au NSDAP. Lui plaide pour l'intégration des anciens nazis qui ne comptaient pas parmi les responsables, afin de ne pas dresser la population contre le nouvel État ${ }^{(28)}$. Ici aussi, il parvient par conséquent à infléchir une position de l'espace public français dans l'intérêt de l'espace public allemand. Par ailleurs, il ne pense pas qu'il est possible d'appréhender la dénazification par une procédure juridique à la manière des Américains, il lui préfère une

21 «Anerkennung, daß bei der Militärregierung die oberste Gewalt liegt, daß dies aber die Verantwortlichen unseres Landes nicht von der Pflicht entbindet, bei Gefährdung der Lebensinteressen der [...] Bevölkerung sowohl speziellen Anordnungen als auch der allgemeinen Politik der Besatzungsmacht gegenüber Widerstand zu leisten », C. Schmid, Erinnerungen (note 6), p. 239.

22 Ibid., p. 238.

23 E. Wolfrum, Französische Besatzungspolitik und deutsche Sozialdemokratie (note 7), p. 178.

24 H. Auerbach, «Die politischen Anfänge Carlo Schmids» (note 2), p. 603.

25 E. Wolfrum, Französische Besatzungspolitik und deutsche Sozialdemokratie (note 7), p. 179.

26 P. WeBer, Carlo Schmid: 1896-1979 (note 3), p. 225 et 224.

27 E. Wolfrum, Französische Besatzungspolitik und deutsche Sozialdemokratie (note 7), p. 206-207.

28 P. Weber, Carlo Schmid: 1896-1979 (note 3), p. 231. 
procédure administrative, comme l'occupant français ${ }^{(29)}$. Mais ce dossier figure l'une des multiples illustrations de l'anéantissement de la collaboration entre le secrétariat d'État et le gouvernement militaire par le commandement central de Baden-Baden: en février 1947, celui-ci impose un système de tribunaux. Schmid est très déçu ${ }^{(30)}$. On constate ainsi que l'espace public allemand pâtit parfois des dissensions perceptibles dans l'espace public français.

En sa qualité de ministre de l'Éducation, Schmid est guidé par le souhait d'instaurer une pédagogie humaine, propre à familiariser les élèves avec la démocratie ${ }^{(31)}$. Tous ses idéaux ne furent pas réalisés, en dépit d'une grande cohésion entre sa vision et celle du gouvernement militaire, quant à «la priorité de la politique d'éducation, le renouveau de la formation des professeurs, [...] la formation des élites et la concentration des programmes sur la formation philosophique et littéraire ${ }^{(32)}$. Dans un discours, Schmid affirme: «Là où il s'agissait de faire quelque chose pour les écoles du Wurtemberg, le gouvernement français ne nous a jamais refusé les autorisations nécessaires [...]» ${ }^{(33)}$. Néanmoins, les vues de Schmid et de l'occupant diffèrent sur quelques points. Tout en reconnaissant qu'étant donné que l'«école est pénétrée de la détermination chrétienne de la culture occidentale», il n'est pas favorable à l'école libre qui «rend difficile l'éducation du peuple à la tolérance envers des opinions allogènes » ${ }^{(34)}$. Cependant, il ne peut imposer son concept et espère en vain le soutien des Français. Toutefois, Schmid s'allie à une occasion avec l'Église catholique contre le gouvernement militaire, lorsque celui-ci envisage de réduire l'enseignement du latin et du grec au profit du français. Les Français finissent par céder aux protestations conjuguées. Ils s'imposent en revanche sur les manuels que Schmid juge inaptes à éduquer à la démocratie, car conduisant trop à penser à la manière française par l'inculcation du sens de l'autorité et de la hiérarchie ${ }^{(35)}$. Dans ce dossier, on observe une combinaison de collaboration et de confrontation entre les agents des deux espaces publics.

Nommé par l'université de Tübingen, en mai 1945, médiateur entre l'université et l'occupant, Schmid devient bientôt représentant des enseignants au sénat. En tant que ministre de l'Éducation, il entreprend la démocratisation et l'épuration de l'université très contaminée par le national-socialisme, mais s'il arrive à faire élire un président selon ses vœux et à restaurer la constitution supprimée en 1933, l'université s'oppose à l'exclusion de professeurs compromis ${ }^{(36)}$. Le gouvernement français promulgue

29 E. Wolfrum, Französische Besatzungspolitik und deutsche Sozialdemokratie (note 7), p. 207.

30 P. Weber, Carlo Schmid: 1896-1979 (note 3), p. 233.

31 G. Hirschler, Carlo Schmid und die Gründung der Bundesrepublik (note 1), p. 62.

32 «Priorität der Bildungspolitik [...], Erneuerung der Lehrerausbildung, Förderung der Elitebildung, Konzentration der Lehrpläne auf philosophische und literarische Bildung», P. WEBER, Carlo Schmid: 1896-1979 (note 3), p. 243.

33 «Wo immer es sich darum handelte, etwas für die Schulen Württembergs zu tun, hat die französische Militärregierung uns nie die erforderlichen Genehmigungen versagt [...]», Karl ScHMID, «Die Aufgaben der Schule», in: K. Schmid, Die Forderung des Tages (note 18), p. 104.

34 Rundfunkansprache von Herrn Prof. Dr. Sснмıd anläßlich des Wahlkampfes Mai 1947, Archiv der sozialen Democratie (AdsD), 1/CSAA000079, p. 2.

35 P. Weber, Carlo Schmid: 1896-1979 (note 3), p. 246-247.

36 Alfred GeIsel, "Carlo Schmid. Gründer der SPD in Württemberg-Hohenzollern und seine Beziehung zur Universität Tübingen», in: G. TAdDEy (dir.), Carlo Schmid (note 11), p. 27. 
un ordre d'épuration conforme aux prescriptions de Schmid ${ }^{(37)}$, qui se souvient que l'officier en charge de l'éducation, René Cheval, lui fut d'un grand soutien, acceptant notamment en 1946 son refus de bloquer l'accès de l'université à certains jeunes en provenance de maisons d'éducation nationales-socialistes ou responsables dans des organisations de la jeunesse ${ }^{(38)}$. Le secrétariat d'État nomme Schmid professeur en avril 1946, malgré les préventions du sénat de l'université( ${ }^{(39)}$. On peut conclure ici à une parfaite cohésion entre les desiderata en provenance de l'espace public allemand et les décisions entérinées par l'espace public français.

En automne 1946, l'élection des assemblées de districts donne la majorité absolue à la CDU, insuffisamment représentée au secrétariat d'État. Schmid se prononce auprès du gouvernement militaire pour un remaniement ministériel. Il reste président et ministre de la Justice, mais doit renoncer à l'Éducation ${ }^{(40)}$. En l'absence de représentation parlementaire jusqu'à l'élection du Landtag, Schmid a su créer un organe de contrôle quasiparlementaire en réunissant régulièrement les sous-préfets (Landräte). Lors d'une session, il salue un représentant du gouvernement militaire ${ }^{(41)}$. Il lui revient la tâche ingrate d'engager les sous-préfets à mettre en œuvre des mesures peu populaires du secrétariat d'État, en réalité des directives de l'occupant. Cependant, les sous-préfets figurent plutôt un soutien au secrétariat d'État contre celui-ci et, lors des négociations avec le gouvernement militaire, Schmid peut se référer aux interpellations des sous-préfets ${ }^{(42)}$. On remarque dans ce dossier que les représentants de l'espace public allemand s'entendent à faire front face aux exigences posées par ceux de l'espace public français.

\section{Tentative de conciliation des intérêts des deux espaces publics}

\section{Des rapports étroits avec les autorités militaires françaises}

De manière générale, Schmid s'évertue à articuler dans le sens d'un fonctionnement sans heurts les positions des deux espaces publics, dans l'intérêt de l'espace public allemand, mais il n'y parvient pas toujours. En tant que chef de gouvernement, il est tenu à des contacts constants avec la puissance d'occupation. Les rencontres entre les ministres et l'administration militaire font bientôt place à une représentation du secrétariat d'État par le seul Schmid, une fois par semaine, auprès de Widmer ou de Corbin de Mangoux, ce qui rend les interprètes superflus ${ }^{(43)}$. Entre l'Allemand possédant parfaitement la langue et la culture françaises et les deux Français s'épanouit bientôt un solide rapport de confiance, ce qui tient en partie à la personnalité du gouverneur, un administrateur et non un militaire, qui manifeste une attitude libérale également adoptée par son directeur de cabinet ${ }^{(44)}$. Les trois hommes s'entretiennent aussi de

37 P. Weber, Carlo Schmid: 1896-1979 (note 3), p. 198.

38 C. SCHMID, Erinnerungen (note 6), p. 260.

39 P. Weber, Carlo Schmid: 1896-1979 (note 3), p. 239.

40 G. Hirschler, Carlo Schmid und die Gründung der Bundesrepublik (note 1), p. 63.

41 K. Schmid, "Gedanken zum Aufbau der Verwaltung» (note 18), p. 85.

42 P. Weber, Carlo Schmid: 1896-1979 (note 3), p. 226.

43 H. Auerbach, «Die politischen Anfänge Carlo Schmids» (note 2), p. 604.

44 Hellmuth Auerbach, "Carlo Schmid und die französische Besatzungsmacht», in: G. Taddey (dir.), Carlo Schmid (note 11), p. 32. 
littérature, d'art et de philosophie ${ }^{(45)}$. On observe donc chez les acteurs de l'espace public français autant que chez celui de l'espace public allemand des prédispositions favorables à l'établissement d'une coopération fructueuse et dont profite Schmid pour arracher des avantages pour l'espace public allemand. Habilement, il soustrait en effet des pans de responsabilité gouvernementale aux Français et transfère des tâches sous compétence allemande ${ }^{(46)}$. Le style de gouvernement autoritaire relevant d'une « démocratie éclairée» qu'il prise correspond à la mentalité des fonctionnaires français formés dans la tradition d'un État fort ${ }^{(47)}$. Il partage également le souhait de ces derniers de préalablement éduquer les Allemands à la démocratie ${ }^{(48)}$. En octobre 1946 est inauguré à Schömberg, où les nazis avaient installé une antenne du camp de concentration de Natzweiler, un cimetière pour les victimes de la terreur. Schmid s'incline devant le général Kœnig, le gouverneur militaire de la zone d’occupation française, et reconnaît la culpabilité des Allemands. La photo est commentée fielleusement: Schmid, l'homme qui se soumet à la puissance d'occupation ${ }^{(49)}$.

Il déclare notamment: «Je ne peux que louer le gouvernement militaire français dans notre zone. Dans le cadre des possibilités existantes, ses représentants sont [...] disposés à offrir une collaboration constructive [...] ${ }^{(50)}$. Il montre de surcroît beaucoup de compréhension pour les priorités des Français: il juge «légitime» qu'ils se préoccupent en premier lieu de leurs propres intérêts ${ }^{(51)}$ et comprend qu'ils utilisent le gage que représente la zone d'occupation pour empêcher que soit générée une Allemagne trop puissante ${ }^{(52)}$. Il estime toutefois qu'ils doivent concevoir qu'il est absurde de vouloir dénier aux Allemands le droit de se constituer en État ${ }^{(53)}$. D’ailleurs, il se souvient que le gouvernement militaire favorisa peu à peu un modus vivendi dans lequel l'administration «directe» se réduisit à une simple supervision. Selon lui, cette évolution ressortait à la conclusion qu'il est difficile d'administrer un pays étranger dans les moindres détails et que l'on ne peut convaincre des personnalités autochtones d'assumer des responsabilités qu'à condition de ne pas les régenter ${ }^{(54)}$.

Ce tableau suggère l'image d'une cohérence parfaite entre les deux espaces publics. Cependant, ces contacts entre Schmid, agent de l'espace public allemand, et la puissance d'occupation, agent de l'espace public français, ne se passent pas sans frictions. Lors de ses rencontres avec Widmer et Corbin de Mangoux, il établit un rapport sur les activités du secrétariat d'État et est informé des missions dont il est chargé. Ces

45 P. Weber, Carlo Schmid: 1896-1979 (note 3), p. 220.

46 H. Auerbach, «Die politischen Anfänge Carlo Schmids» (note 2), p. 605-606.

47 H. Auerbach, «Carlo Schmid und die französische Besatzungsmacht» (note 44), p. 31.

48 P. Weber, Carlo Schmid: 1896-1979 (note 3), p. 221.

49 Ibid., p. 252.

50 «Ich kann die französische Militärreg in unserer Zone nur loben. Im Rahmen der gegebenen Möglichkeiten sind ihre Vertreter [...] bereit, [...] konstruktive Arbeit zu leisten ", cité d'après H. AuERBACH, «Die politischen Anfänge Carlo Schmids» (note 2), p. 609-610.

51 Karl SchміD, «Vaterländische Verantwortung», in: K. Schмid, Die Forderung des Tages (note 18), p. 16 .

52 Carlo Schmid, Der Weg des deutschen Volkes nach 1945, Berlin (Ouest), Haude \& Spene, 1967, p. 32.

53 Cité d'après H. Auerbach, «Die politischen Anfänge Carlo Schmids» (note 2), p. 645.

54 C. Schmid, Erinnerungen (note 6), p. 241. 
entretiens se déroulent dans une atmosphère glacée, bien que les officiers français évitent les humiliations directes. Souvent, il doit faire son exposé debout et il est parfois réveillé en pleine nuit pour fournir une information. Schmid, qui déteste les vexations, a bien du mal à avaler ces couleuvres. Ses collègues et lui seraient traités comme des "gamins», s'emporte-t-il, et sa fonction s'apparente souvent à une «marche à travers mille humiliations ${ }^{(55)}$. Par ailleurs, les interventions du gouvernement militaire s'accroissent, le commandement central de Baden-Baden réprouvant la modération de l'administration militaire du Wurtemberg-Hohenzollern. Le secrétariat d'État doit selon le commandement central se borner à être la puissance exécutive du gouvernement militaire ${ }^{(56)}$. Par conséquent, en partie parce que les divergences de vues au sein de l'espace public français nuisent aux intérêts de l'espace public allemand, les rapports entre deux espaces publics ne sont pas exempts de tensions. En outre, Schmid estime frustrant de devoir attirer l'attention des Français sur les limites des livraisons d'aliments ${ }^{(57)}$ et des réquisitions et de devoir ainsi endosser le rôle ingrat du quémandeur. À la longue, sa méthode porte ses fruits. Les Français voient en lui « un excellent comédien ", disposé à user de toutes sortes de ruses pour parvenir à ses fins ${ }^{(58)}$.

Et ceci débouche parfois sur une véritable mise en cause de l'espace public français par l'agent de l'espace public allemand: comme Schmid constate que les Américains ont entrepris la reconstruction du pays, alors que les Français persistent dans son exploitation, il ne se prive pas de critiquer l'occupant. En octobre 1946, il indique que, du fait que le gouvernement de Wurtemberg-Hohenzollern n'est responsable devant personne d'autre que le gouvernement militaire, il ne peut être question de démocratie, fiction entretenue par les occupants. Le commandement à Baden-Baden est indigné. Schmid formule ces blâmes, car il redoute que les hommes politiques allemands qui assument des responsabilités sous l'occupation ne soient tenus pour des «valets du vainqueur ${ }^{(59)}$. En novembre, il donne publiquement à entendre que l'occupation se réduit à une exploitation, adressant néanmoins ses doléances à Baden-Baden et remerciant les officiers du Wurtemberg-Hohenzollern. Le lendemain, il est cité à comparaître à Baden-Baden devant le général Laffon, qui exige qu'il expose publiquement les statistiques françaises concernant le bilan de l'alimentation. Schmid sort renforcé de cette crise, gagnant en renom du fait de sa confrontation avec l'occupant ${ }^{(60)}$. On constate ici un renforcement de la position de Schmid dans l'espace public allemand du fait de sa résistance à la toute-puissance de l'espace public français et, par ailleurs, l'émergence d'un véritable divorce entre les deux espaces publics, qui, paradoxalement, se produit parallèlement à une collaboration incontestable. La présidence du secrétariat d'État de Schmid arrive à échéance avec les élections au Landtag de mai 1947, qui donnent la majorité absolue à la CDU. Membre du SPD, Schmid reste ministre de la Justice

55 «Einem Gang durch tausend Erniedrigungen», P. Weber, Carlo Schmid: 1896-1979 (note 3), p. 229 et 228 .

56 H. Auerbach, «Carlo Schmid und die französische Besatzungsmacht» (note 44), p. 33.

57 Les Allemands devaient livrer aux occupants français des quotas d'aliments pour l'approvisionnement des troupes d'occupation.

58 P. Weber, Carlo Schmid: 1896-1979 (note 3), p. 229-230.

59 «Knechte der Sieger», ibid., p. 254 et 221.

60 Ibid., p. 255-256. 
et devient vice-président, ce qui lui permet de garder en partie les rênes du pouvoir, même si les Français auraient préféré le garder comme président ${ }^{(61)}$.

\section{Des dossiers politiques annexes}

Les Américains chargent en février 1946 Reinhold Maier, le ministre-président de Wurtemberg-Bade, de réunir une commission afin d'ébaucher une constitution. Schmid est convié à se joindre à celle-ci en qualité d'expert. Ses connaissances en matière de droit constitutionnel, sa formation humaniste et son engagement pour le droit et la justice lui confèrent une position privilégiée et le projet de constitution est en grande partie issu de sa main. Il accorde la priorité aux valeurs humaines, à la dignité de l'homme, à la justice sociale, à la démocratie, au rejet de la toute-puissance de l'État et au renoncement à la guerre ${ }^{(62)}$. Dès fin 1946, le gouverneur Widmer a prié Schmid de lui faire des propositions pour l'adoption de la constitution de Stuttgart par le Wurtemberg-Hohenzollern, mais le représentant de la CDU déclare qu'il jugerait le transfert «déshonorant». Toutefois, le projet de la CDU dépasse les pires craintes de Schmid; il est d'ailleurs refusé par le gouvernement militaire comme trop confessionnel, autoritaire et non démocratique. La commission produit un compromis qui ne satisfait personne ${ }^{(63)}$. Une fois encore, on note une intervention des acteurs de l'espace public français à l'adresse de ceux de l'espace public allemand, en faveur de Schmid et en défaveur de la CDU.

Une autre thématique préoccupe Schmid: l'Europe. Comme Churchill, il considère l'antagonisme franco-allemand comme le problème européen cardinal ${ }^{(64)}$. Il juge que sa résolution ne peut être que le produit de l'Europe et non sa condition préalable ${ }^{(65)}$. Ce faisant, il a su comme aucun autre Allemand se mettre à la place des Français, étant d'avis que les Allemands doivent consentir à un contrôle international de l'industrie de la Ruhr ${ }^{(66)}$. «Les Allemands ont le sentiment que les Français font tout pour leur barrer la route d'une nouvelle existence nationale [...]», écrit-il. «Les Français n'agissent pas ainsi pour nous porter préjudice, mais bien parce qu'ils sentent la nécessité d'assurer leur sécurité. [...] L'histoire nous apprend, disent-ils, qu’un État allemand centralisé est un danger qui nous a plusieurs fois presque anéantis » ${ }^{(67)}$. Il propose à ses compatriotes de «contribuer à l'accélération de la construction des États-Unis d'Europe par le choix de notre comportement à l'égard du point le plus faible de l'Europe, à savoir les relations [...] entre le peuple français et le peuple allemand ${ }^{\left({ }^{(68)}\right.}$. On peut conclure

61 G. Hirschler, Carlo Schmid und die Gründung der Bundesrepublik (note 1), p. 65.

62 Paul SAUER, "Carlo Schmid und die Entstehung der Verfassung von Württemberg-Baden», in: G. TADDEY (dir.), Carlo Schmid (note 11), p. 61-62.

63 P. Weber, Carlo Schmid: 1896-1979 (note 3), p. 279-281.

64 Edgar Wolfrum, «Deutschland, Frankreich, Europa - frühe europapolitische Pläne Carlo Schmids », in: G. TAdDey (dir.), Carlo Schmid (note 11), p. 45.

65 Carlo Schmid, «France-Allemagne-Europe», Deutsche Rundschau, 3.03.1949, AdsD, 1/CSAA000084, p. 9.

66 E. Wolfrum, «Deutschland, Frankreich, Europa» (note 64), p. 52.

67 C. Scнмid, «France-Allemagne-Europe» (note 65), p. 5.

68 «Durch die Art unseres Verhaltens gegenüber dem wundesten Punkt Europas, nämlich dem [...] Verhältnis des deutschen und des französischen Volkes, einiges zur Beschleunigung des Werdeprozesses 
ici à une empathie d'un représentant de l'espace public allemand envers l'espace public français et à une reconnaissance des motivations de ce dernier. Schmid souhaite pour l'Europe un schéma constitutionnel fédéral: "Il faut donner [à l'Europe]», écrit-il, «une unité économique, politique, constitutionnelle. Elle ne doit pas être une large confédération d'états, il faut un véritable état fédéral » ${ }^{(69)}$. À l'origine, Schmid, pour qui la question allemande et l'Union européenne sont indissolublement liées, s'oppose à l'intégration à l'Ouest et se prononce pour un État provisoire, mais suite à la formation des deux blocs, il change radicalement d'avis ${ }^{(70)}$.

\section{Au sein du SPD}

Alors que les Américains accordent des licences aux partis dès août 1945, la zone française doit attendre jusqu'en janvier 1946. Il faut au préalable obtenir l'aval du secrétariat d'État pour fonder un parti, ce qui convient à Schmid ${ }^{(71)}$. Pour sa part, convaincu qu'il ne peut se révéler efficace qu'au sein d'un parti, il se tourne vers le SPD du fait de son fondement humaniste et social ${ }^{(72)}$. Ce qui le convainc n'est néanmoins pas la force de l'analyse marxiste, mais la volonté d'un engagement pour les défavorisés. Il vient à la social-démocratie moins par Marx, qui voulait faire disparaître l'État, que par Ferdinand Lassalle, partisan d'un État fort ${ }^{(73)}$. Dès l'arrivée des troupes d'occupation, il a pris contact avec d'anciens membres du SPD et prend en main la refondation du parti. Il fait office de président régional avant même son élection. Beaucoup se rallient au parti suite à sa promesse de transformer le SPD en parti de masse, et on note qu'il n'y a aucune trace de marxisme dans le programme que Schmid présente en février 1946 au gouvernement militaire. Un mois plus tard, il obtient la licence ${ }^{(74)}$. Les Français, qui soulignent que la social-démocratie du SudOuest s'arrange le mieux de la politique de démocratisation française ${ }^{(75)}$, tolèrent les voyages de Schmid à l'occasion de congrès ${ }^{(76)}$, reconnaissant en lui un homme à même d'engager l'évolution doctrinale du SPD. Cela les conduit à des jugements erronés : à partir de 1947, ils considèrent Schmid comme l'adversaire et le dauphin d'un Kurt Schumacher nationaliste et unitariste. En outre, ils apprécient sa capacité à combattre les tendances nordistes et prussiennes du parti ${ }^{(77)}$. Ainsi, même dans ce domaine qui n'entre pas dans le champ de leurs compétences, les agents de l'espace public français soutiennent l'agent de l'espace public allemand.

der Vereinigten Staaten von Europa beitragen », Karl SснміD, «Das deutsch-französische Verhältnis und der dritte Partner», Die Wandlung, Jahrgang II, Heft 9 (1947), p. 800.

C. Schmid, «France-Allemagne-Europe» (note 65), p. 8.

70 E. Wolfrum, «Deutschland, Frankreich, Europa» (note 64), p. 53.

71 P. Weber, Carlo Schmid: 1896-1979 (note 3), p. 261.

72 A. GeIsel, «Carlo Schmid. Gründer der SPD in Württemberg-Hohenzollern und seine Beziehung zur Universität Tübingen» (note 36), p. 23.

73 E. Wolfrum, Französische Besatzungspolitik und deutsche Sozialdemokratie (note 7), p. 113.

74 P. Weber, Carlo Schmid: 1896-1979 (note 3), p. 261-262 et 265.

75 E. Wolfrum, Französische Besatzungspolitik und deutsche Sozialdemokratie (note 7), p. 17.

76 P. WeBer, Carlo Schmid: 1896-1979 (note 3), p. 271.

77 E. Wolfrum, Französische Besatzungspolitik und deutsche Sozialdemokratie (note 7), p. 119 et 294. 


\section{De l'espace public régional à l'espace public national - le divorce avec l'espace public français}

Il était prévisible que Schmid ne bornerait pas ses activités au Wurtemberg-Hohenzollern et aspirerait, voire serait appelé à étendre son rayon d'action à l'espace public allemand au niveau national. Toutefois, ses ambitions se heurtent à la réticence de la puissance d'occupation à autoriser les responsables de sa zone à participer à des actions à l'extérieur. Ici, les ambitions et intérêts des représentants des deux espaces publics divergent. La première institution de la bizone à laquelle Schmid s'associe à partir d'avril 1947 est le Bureau allemand pour les questions de paix (Deutsches Büro für Friedensfragen), précurseur d'un ministère des Affaires étrangères. Néanmoins, il doit rendre sa participation plus ou moins confidentielle à cause des Français ${ }^{(78)}$. Schmid est un fédéraliste convaincu, et les Français le considèrent à ce titre comme l'adversaire de Schumacher. Cependant, ils n'ignorent pas que sa conception ne correspond pas vraiment à la leur: prônant la création d'un État «fédératif» et non "fédéraliste», il est partisan d'un État fédéral doté de compétences étendues ${ }^{(79)}$.

Il travaille aussi à l'élaboration du nouvel État dans un autre cadre: le ministreprésident de Bavière Hans Ehard convie les ministres-présidents de toutes les zones à une conférence pour juin 1947. Pour arracher à l'occupant l'autorisation d'y assister, Schmid argue qu'en l'absence des chefs de gouvernement de la zone française, les tendances centralistes pourraient s'exprimer trop massivement. Les Français se laissent convaincre, mais interdisent toute participation à des débats politiques et juridiques. Le 6 juin, Schmid livre un bilan de l'alimentation catastrophique en zone française, des quotas de livraison d'aliments élevés et des réquisitions dommageables pour l'agriculture $^{(80)}$. Il lui tient aussi à cœur de faire voter un statut de l'occupation: lors de la conférence, le 7 juin, il affirme: « [...] Dans le domaine de la législation, de l'administration et de la jurisprudence doit s'effectuer une délimitation exacte des compétences des instances allemandes et alliées. [...] Elle doit s'effectuer d'une certaine manière, c'est-à-dire de façon à ce que toutes les matières qui ne relèvent pas directement des objectifs [...] de l'occupation soient déclarées de l'exclusive compétence des instances allemandes ${ }^{(81)}$. Il ajoute que pour l'approvisionnement des troupes d'occupation ne devrait être prélevée que la quantité d’aliments garantissant les calories nécessaires pour la population civile ${ }^{(82)}$. Plus tard, il mentionnera trois justifications de ce statut: sans lui, il est premièrement impossible d'administrer les Länder; deuxièmement, il est impossible de les gérer financièrement si les coûts de l'occupation ne sont pas fixés à long terme; troisièmement, il est impossible de diriger la répartition des biens de

78 G. Hirschler, Carlo Schmid und die Gründung der Bundesrepublik (note 1), p. 101 et 103.

79 P. Weber, Carlo Schmid: 1896-1979 (note 3), p. 286-287.

80 G. Hirschler, Carlo Schmid und die Gründung der Bundesrepublik (note 1), p. 113-114 et 117.

81 «[...] auf dem Gebiete der Gesetzgebung, der Verwaltung und Rechtsprechung [muß] eine genaue Abgrenzung der Befugnisse der deutschen und der alliierten Stellen erfolgen. [...] sie muß in einer bestimmten Weise erfolgen, nämlich so, daß alle Materien, die nicht unmittelbar zu den [...] Besatzungszwecken gehören, zur ausschließlichen Kompetenz der deutschen Stellen erklärt werden müssen», Carlo SCHмID, «Über die Notwendigkeit eines Besatzungsstatuts», AdsD, 1/CSAA000081, p. 3.

82 Carlo Schмid, "Ansprache von Herrn Staatsrat Prof. Dr. ScHмid anläßlich der Ministerpräsidentenkonferenz in München im Juni 1947 über die Regelung des Besatzungsrechtes », AdsD, 1/CSAA002231, p. 5-6. 
consommation si l'on ne connaît pas la quantité des prélèvements ${ }^{(83)}$. À Munich, une résolution pour la conclusion d'un statut de l'occupation est votée, et Schmid travaille à l'élaboration d'un statut dans le cadre d'une commission au sein du Bureau allemand pour les questions de paix ${ }^{(84)}$. Cependant, le statut promulgué en juillet 1948 par les Alliés présente des accents plus généraux et régaliens ${ }^{(85)}$.

Au plus tard lors de cette conférence, Schmid a acquis le renom d'un homme politique prometteur au-delà de l'espace public du Wurtemberg, intéressant pour la presse, une popularité qui lui est bien utile, car ses exposés ont indigné l'occupant français ${ }^{(86)}$. Plus que jamais, celui-ci le tient pour le dauphin de Schumacher, à même de conférer au SPD une orientation moins nationaliste et plus européenne. Schmid commence à laisser filtrer qu'il pourrait quitter la zone française, ce qui effraie la puissance d'occupation qui ne s'entend guère avec le nouveau chef du gouvernement Laurenz Bock ${ }^{(87)}$. Du côté français, on est satisfait du déroulement de la conférence et convaincu que le ministre-président de Bavière n'a pu imposer le principe fédéraliste qu'avec le soutien des représentants de la zone française, et en particulier Schmid ${ }^{(88)}$. On observe donc, en conclusion de la conférence de Munich, un rapprochement entre les acteurs des deux espaces publics, qui se confirme par la suite: l'occupant français atermoie longtemps devant la perspective de la trizone, et Schmid, qui pousse dans ce sens, mène de longues discussions à ce sujet avec le général Kœnig, à l'initiative de celui-ci, avant que les Français ne se décident en avril $1949^{(89)}$.

En tout état de cause, la conférence de Munich semble avoir fait évoluer la position du haut commandement français envers Schmid: l'ambassadeur Tarbé de Saint-Hardoin écrit à Paris qu'on devrait fournir plus souvent à celui-ci l'occasion d'intervenir en dehors de la zone française. Néanmoins, le Quai d'Orsay, méfiant, veut d'abord lui interdire de participer au congrès du SPD de Nuremberg en juin 1947, mais change d'avis suite à l'intervention de Saint-Hardoin, qui fait valoir que son absence renforcerait les forces anti-françaises et centralistes. Schmid est élu au sein du comité central du parti et plaide résolument pour une structure fédérale du nouvel État ${ }^{(90)}$. Un rapport de la Sûreté nationale française indique: «Certes, Schmid est profondément allemand; mais on ne lui ferait pas de tort en le qualifiant de "collaborateur" de grande classe, qui s'entend habilement à équilibrer les intérêts de son pays et ceux de la France » ${ }^{(91)}$. On ne saurait imaginer attestation plus convaincante de la capacité de Schmid à concilier les positions des deux espaces publics. Après la formation du gouvernement parlemen-

83 Carlo Schмid, «Diskussion am runden Tisch über das Thema Besatzungsstatut am 19.02.1948 im Nordwestdeutschen Rundfunk», AdsD, 1/CSAA000080.

84 G. Hirschler, Carlo Schmid und die Gründung der Bundesrepublik (note 1), p. 125.

85 H. Auerbach, «Die politischen Anfänge Carlo Schmids» (note 2), p. 643.

86 G. Hirschler, Carlo Schmid und die Gründung der Bundesrepublik (note 1), p. 118.

87 P. WeBer, Carlo Schmid: 1896-1979 (note 3), p. 299-300.

88 H. Auerbach, «Die politischen Anfänge Carlo Schmids» (note 2), p. 629.

89 C. SCHMid, Erinnerungen (note 6), p. 307.

90 H. Auerbach, «Die politischen Anfänge Carlo Schmids» (note 2), p. 630-632.

91 «Sicher ist Schmid zutiefst Deutscher; aber man würde ihm nicht Unrecht tun, wenn man ihn als "Kollaborateur" großer Klasse bezeichnet, der auf geschickte Weise die Interessen seines Landes und die Frankreichs auszugleichen versteht», ibid., p. 635. 
taire en juillet 1947, Schmid n'est plus le premier homme du Land, mais en qualité de suppléant du ministre-président, il fait office de «ministre des Affaires étrangères » du Land et continue de le représenter à Stuttgart et lors des conférences des Länder des trois zones occidentales. Le chef du gouvernement Bock le charge des négociations avec la puissance d'occupation ${ }^{(92)}$. Cependant, Schmid, élu à l'automne 1949 député au Bundestag, se retire peu à peu du Land, même s'il conserve son poste de ministre de la Justice jusqu'au printemps 1950.

Ce furent les enjeux de l'occupation française qui firent de Schmid un homme politique en 1945. Et ce fut son mérite de permettre à l'administration du WurtembergHohenzollern de s'imposer comme force politique autonome, c'est-à-dire de permettre à l'espace public allemand de faire valoir ses intérêts face à l'espace public français. Il y parvient par ses efforts incessants pour rendre compatibles autant que faire se peut les intérêts des deux espaces publics, tâche qui lui est facilitée par l'a priori favorable des Français à son égard: ceux-ci reconnaissaient en lui un homme politique de valeur, qui, en dépit de sa critique des effets de l'occupation, était plus compréhensif envers leurs intérêts que la plupart des hommes politiques de l'époque ${ }^{(93)}$. Schmid acquit rapidement la carrure d'une des personnalités les plus marquantes de la zone d'occupation et les Français lui accordèrent moult droits particuliers.

Quelques réflexions pour conclure sur l'existence ou non à cette époque d'un espace public transnational - ici, plus exactement binational - germanophone. Dans le Wurtemberg se produit alors une confrontation de deux espaces publics ou plus exactement une irruption de l'espace public de l'occupant, c'est-à-dire français, dans l'espace public allemand. Schmid lui-même, du fait de ses origines binationales, participe d'un espace public transnational. N’est-il pas désigné par ses adversaires comme «l'homme des Français »? Il s'avère à la fois la cheville ouvrière et le vecteur des transferts, c'està-dire un médiateur, souvent critique, entre les deux espaces publics. Ainsi, l'espace de débats présidant à l'édification du nouvel État se révèle le fruit de la collaboration, voire de la confrontation des deux espaces publics. Dans cette optique, nous avons analysé comment les représentations françaises ont pesé sur l'élaboration du système politique et social allemand. Un bon exemple est constitué par l'éducation, une priorité pour l'occupant comme pour Schmid; un autre le système de dénazification; ou encore la constitution. Concernant ces trois dossiers, on observe une convergence des vues des Allemands et du gouvernement militaire, qui se retrouve dans la prédilection pour un style de gouvernement autoritaire. On note ici une cohésion notable des deux espaces publics, d'autant que Schmid atteste beaucoup de compréhension pour le besoin de sécurité des Français. Dans le même ordre d'idées, les Français considèrent Schmid comme l'adversaire et le dauphin de Kurt Schumacher, à même d'engager une évolution du SPD dans une direction moins nationaliste et unitariste.

Cependant, l'occupant français est mû par la volonté d'empêcher le nouvel État de se constituer de telle manière qu'il puisse nuire de nouveau à la France par une agression. Dans cette perspective, nous avons constaté que l'espace public allemand se prémunit aussi de l'intrusion de l'espace public français et développe, sous la houlette 
de Schmid, ses propres conceptions. Celui-ci s'efforce sans relâche d'élargir sa marge de manœuvre et d'éviter que le secrétariat d'État se réduise à une administration obtempérant aux injonctions du gouvernement militaire. Ceci est souvent toléré par l'occupant, qui comprend que l'on ne peut administrer un pays dans ses moindres détails. Il arrive également que les deux espaces publics, en la personne du gouvernement militaire et de Schmid, s'affrontent sans compromis possible. C'est le cas à propos des démontages et des livraisons de nourriture, que Schmid juge ressortir à l'exploitation, ou du statut de l'occupation qu'il exige. Et cette combinaison de collaboration et de confrontation entre les deux espaces publics dépasse le cadre du Wurtemberg, puisqu'après des réticences, les Français accompagnent le passage de Schmid à un niveau de responsabilité nationale. On peut même affirmer que ce processus vaut pour toute l'Allemagne, puisqu'elle est entièrement occupée. Et du fait de la porosité de l'espace public allemand aux représentations des espaces publics des puissances d'occupation, il nous semble qu'on peut conclure pour l'Allemagne d'immédiat aprèsguerre à l'existence d'un espace public transnational.

\section{Résumé}

Né en France de mère française et ayant passé ses premières années en France, Carlo Schmid est parfaitement bilingue, ce qui lui facilite les rapports avec la puissance d'occupation française lorsqu'il occupe des fonctions gouvernementales en Wurtemberg pendant l'immédiat après-guerre. Après avoir dirigé le Bloc antifasciste à Tübingen, il devient, à l'initiative des Français, ministre de l'Éducation à Stuttgart, puis, en Wurtemberg-Hohenzollern, président $d u$ «secrétariat d'État » et ministre du Culte, de l'Éducation et des Arts. En étroite collaboration, mais parfois confrontation, avec l'occupant, auquel il est lié par un solide rapport de confiance, il s'engage dans la politique de dénazification et de l'éducation. Il joue un rôle central dans la rédaction de la constitution de Wurtemberg-Bade, puis de Wurtemberg-Hohenzollern. Il prend en main la refondation du SPD régional. Peu à peu, il élargit sa zone d'action au niveau national. Dans cette irruption de l'espace public français dans l'espace public allemand, on distingue l'émergence d'un espace public transnational.

\section{Zusammenfassung}

In Frankreich von einer französischen Mutter geboren und nach den ersten Lebensjahren in Frankreich, ist Carlo Schmid absolut zweisprachig, was ihm die Beziehungen zur französischen Besatzungsmacht erleichtert, als er in der unmittelbaren Nachkriegszeit Regierungsämter in Württemberg übernimmt. Nachdem er in Tübingen den Antifaschistischen Block geleitet hat, wird er auf Initiative der Franzosen Erziehungsminister in Stuttgart und dann in Württemberg-Hohenzollern Vorsitzender des "Staatssekretariats» und Minister für Justiz, Kult, Erziehung und Kunst. In enger Zusammenarbeit, aber auch Konfrontation, mit der Besatzungsmacht, mit er ein solides Vertrauensverhältnis unterhält, trifft er in der Entnazifizierungs- und Erziehungspolitik wegweisende Maßnahmen. Er spielt bei der Niederschrift der Verfassung von Württemberg-Baden und dann von Württemberg-Hohenzollern eine grundlegende Rolle. Er übernimmt die Neugründung der Landes-SPD. Allmählich dehnt er sein Wirkungsfeld aufBundesebene 
aus. In dieser Einmischung der französischen Öffentlichkeit in die deutsche ist die Entstehung einer transnationalen Öffentlichkeit zu erkennen.

\begin{abstract}
Carlo Schmid, born in France of a French mother and after his first years in France, is absolutely bilingual which facilitates his relationship with the French occupation forces while he occupied government positions in Württemberg during the immediate post war period. Having led the Anti-fascist Bloc in Tübingen, he becomes Education minister in Stuttgart at the impetus of the French and then becomes in Württemberg-Hohenzollern president of the "State secretariat» and minister of cult, education and the arts. In close collaboration, although at times in confrontation with the occupying forces, with whom he enjoys a position of solid trust, he is involved in the denazification and education process. He plays a pivotal role in the drafting of the Württemberg-Baden constitution and subsequently that of Württemberg-Hohenzollern. He is in charge of the re-establishment of the regional SPD. Little by little, he increases his realm of action to the national level. With this sudden emergence of the French public sphere within the German public sphere, the birth of a transnational public sphere can be noted.
\end{abstract}

